\title{
The Roles of Infrastructure and Resources on Implementation of Free Maternal Healthcare Services in Machakos Level 5 Hospital, Machakos County, Kenya
}

\author{
Otundo Denis Orare ${ }^{1, *}$, Wangombe Ann ${ }^{1}$, Muchiri Francis ${ }^{2}$, Chimbevo Mwagambo ${ }^{3}$, \\ Ooga Westley ${ }^{4}$ \\ ${ }^{1}$ Institute of Tropical and Infectious Diseases, College of Health Sciences, University of Nairobi, Nairobi, Kenya \\ ${ }^{2}$ ITROMID, College of Health Sciences, Jomo Kenyatta University of Agriculture and Technology, Nairobi, Kenya \\ ${ }^{3}$ Department of Biochemistry, School of Medicine, Mt. Kenya University, Thika, Kenya \\ ${ }^{4}$ Ministry of Health, Nairobi, Kenya
}

Email address:

denotundo@yahoo.com (O. D. Orare.)

${ }^{*}$ Corresponding author

\section{To cite this article:}

Otundo Denis Orare, Wangombe Ann' Muchiri Francis, Chimbevo Mwagambo, Ooga Westley. The Roles of Infrastructure and Resources on Implementation of Free Maternal Healthcare Services in Machakos Level 5 Hospital, Machakos County, Kenya. Science Journal of Public Health. Vol. 5, No. 1, 2017, pp. 49-55. doi: 10.11648/j.sjph.20170501.18

Received: December 6, 2016; Accepted: December 27, 2016; Published: January 20, 2017

\begin{abstract}
Free maternal health care services in Kenya was introduced on $1^{\text {st }}$ June, 2013 and has increased the number of skilled deliveries significantly. In this paper an attempt is made to examine the factors associated with the utilization of free maternal health care services in Kenya on the basis of data collected from Machakos Level 5 hospital. A descriptive crosssectional study design was used. Using both probability and non-probability sampling techniques; purposive sampling and stratified sampling techniques, a sample of 398 participants who received free maternal services at Machakos level 5 were recruited. A survey questionnaire and interview schedule were administered to women who had delivered in the hospital and interview scheduled was administered to health workers. The findings of the study showed that there is a recognizable relationship between hospital infrastructure and implementation of free maternity services in public hospitals. The study established that there is a positive and significant relationship between patient level of awareness and utilization of free maternal healthcare services. Awareness was sighted as a major structural variable that could influence decision of women to deliver in public hospitals, such knowledge should highlight on requirement and various point of service delivery all of which determined to a great extent the quality of free maternal health services in Kenya.
\end{abstract}

Keywords: Free Maternity Service, Resources, Infrastructure

\section{Introduction}

Over the last two decades, there have been investments in public health system in Kenya with the emphasis on providing maternal healthcare services and at the same time encouraging women to deliver under supervision of skilled healthcare professionals. However, the uptake of maternal healthcare services is very low standing at $43 \%$ of women delivering in hospital [1]. Further, 1 in 25 women have a chance /risk of dying from pregnancy and child birth complication in a life time (KDHS, 2009). Although attending antenatal clinics and skilled personnel is significance in relation to maternal mortality and morbidity, utilization of maternal healthcare services is highly associated with improved maternal health outcomes [2]. Reduction of the risk of maternal morbidity and mortality, especially in places where general health status of a woman is poor effective use of maternal healthcare services is crucial $[2,3]$.

Reducing maternal mortality has seen the government and the international agencies promote maternal healthcare services. Although, reducing maternal mortality requires 
different strategies to promote health of pregnant women. This has elicited reactions with United Nations member countries incorporating maternal health within the Millennium Development Goals (MDGs) target points and the target to reduce maternal mortality and morbidity [4]. In recent years, several African countries have enacted policies to make deliveries and/or health care for mothers and children free or nearly free in order to fulfill these mandates [5]. Kenya's new free maternal health services policy is a potentially positive step in this direction. However, compliance with international, regional, and local obligations, implementation of this policy must not override or diminish other rights provided by these frameworks [6].

Kenya is also committed to improve maternal mortality ratio, one of the MDGs through acts of parliament (Constitution of Kenya, 2010). In efforts to increase Kenyans having access to quality health care, the government raised the budgetary allocation on health from $6 \%$ to $10 \%$ in $2014-2015$ fiscal years, though still lower than the $15 \%$ recommended standard of the national budget that was agreed on in the Abuja Declaration of April 2001 [5]. The government of Kenya and other international agencies join hand and resources to promote maternal healthcare services. Although reducing maternal mortality and morbidity requires different strategies and approach to promoting maternal health.

Given the perspective of poverty and lack of quality maternal healthcare services in Kenya, implementation of free maternal healthcare services will depend on improved hospital infrastructure, increased resources outlay, staffing and improved remuneration packages for medical staffs. Therefore, evaluation of the influence of resources and infrastructure on the implementation of free maternity health care services in Kenya is paramount. The current study seeks to establish the influence of sources of information and patient satisfaction on implementation of free maternal healthcare services in Kenya a case study of Machakos Level 5 Hospital.

Machakos County provides an ideal setup to carry out this research due to the nature and the characteristics of the study population, as foreign and local investors have injected more help to the sector through the ministry of health. The study brings into light the various factors that the investors and the government need to focus on when targeting provision of free maternal healthcare services, form basis of discussion of maternal healthcare services in developing countries, help understand factors influencing and possible alternatives to the health assistance which would assist the positive outcome and identification of individual factors that may influence or impede implementation of free maternal healthcare services in public hospitals.

\section{Methods}

A descriptive cross-sectional study design was employed in this research. The study focused on one referral health facilities in Machakos County (The Machakos Level 5 Hospital) targeting a population of 120,000 women of reproductive age seeking to deliver or post-natal services in Machakos level five hospital from the year 2013 - 2015 as per the recorded figures obtained from DHIS. Using both probability and non-probability sampling techniques; purposive sampling and stratified sampling techniques, a cross sectional study was conducted [7]. A total of 398 women pregnant women was to be selected from the four public hospitals in Machakos County. Qualitative methods were used to complement the quantitative data. A survey questionnaire and interview schedule were administered to women who had delivered in the hospital and interview scheduled was administered to health workers. The data was collected from 2013 to 2015 and analysis of the data was done using SPSS software and Microsoft Office Excel. The degree of association between responses was tested at $95 \%$ confidence interval. Ethical Clearance to carry out the research was obtained from Kenyatta National Hospital (KNH) and University of Nairobi (UON) Board of Scientific and Ethics Review Committee. Authorization to conduct the study at the Machakos level 5 Hospital was given by the Medical Superintendent following perusal and approval by the research committee of the facility. Codes were used to identify the respondents thus withholding the identity of the respondents.

\section{Results}

\subsection{Response Rate}

Out of 398 questionnaires, 324 were filled and returned thus a total 324 respondents participated in the study out of the sampled 398 , giving an overall response rate of $81.4 \%$.

\subsection{Demographic Information of the Respondent}

The demographic information of respondents included age, marital status, education, occupation and average monthly income of women who delivered in public hospitals Machakos level 5 Hospital is presented in Table 1. Majority of the respondent $50.6 \%$ were beneficiary in the age of 21 to 30 years while the least was $2.5 \%$ in the age above 41 years. However, majority of the women $65.4 \%$ were married and in stable relationships and the least $2.5 \%$ and $1.2 \%$ were divorced and widowed respectively. In terms of level of education, $53.1 \%$ of the respondents had secondary education while $4.9 \%$ had no formal education. Majority 164 (50.6\%) of the women who utilized free maternal healthcare services most were self-employed with 128 (39.5\%) having an income of between KSH. 10,001-20,000.

\subsection{Service Charges in Public Hospitals}

The study also aimed to establish whether patients were paying for some services related to maternal healthcare. The results are presented in Table 2. It was identified that Machakos level 5 hospitals was not charging admission fees for women seeking delivery services. However, due to lack of equipment such as obstetric ultra sound, antenatal clinic due to congestion and antenatal profile test were carried outside the hospital at a fee. 
Table 1. Demographic information of the respondents.

\begin{tabular}{|c|c|c|c|c|}
\hline Demographic parameter & Frequency & $\%$ Frequency & $\%$ Validity & $\%$ Cumulative \\
\hline \multicolumn{5}{|l|}{ Age } \\
\hline Below 20 years & 72 & 22.2 & 22.2 & 22.2 \\
\hline $21-30$ years & 164 & 50.6 & 50.6 & 72.8 \\
\hline $31-40$ years & 80 & 24.7 & 24.7 & 97.5 \\
\hline $41-50$ years & 8 & 2.5 & 2.5 & 100.0 \\
\hline \multicolumn{5}{|l|}{ Marital Status } \\
\hline Single & 100 & 30.9 & 30.9 & 30.9 \\
\hline Married & 212 & 65.4 & 65.4 & 96.3 \\
\hline Divorced & 8 & 2.5 & 2.5 & 98.8 \\
\hline Widowed & 4 & 1.2 & 1.2 & 100.0 \\
\hline \multicolumn{5}{|l|}{ Level of education } \\
\hline None & 16 & 4.9 & 4.9 & 4.9 \\
\hline Primary & 36 & 11.1 & 11.1 & 16.0 \\
\hline Secondary & 172 & 53.1 & 53.1 & 69.1 \\
\hline College & 100 & 30.9 & 30.9 & 100.0 \\
\hline \multicolumn{5}{|l|}{ Occupations } \\
\hline Unemployed & 64 & 19.8 & 20.8 & 20.8 \\
\hline Self employed & 164 & 50.6 & 53.2 & 74.0 \\
\hline Formally employed & 80 & 24.7 & 26.0 & 100.0 \\
\hline No response & 16 & 4.9 & 100.0 & \\
\hline \multicolumn{5}{|l|}{ Average Monthly Income } \\
\hline Below Ksh.10 000 & 64 & 19.8 & 25.8 & 25.8 \\
\hline Ksh. $10001-20000$ & 128 & 39.5 & 51.6 & 77.4 \\
\hline Ksh. $20001-30000$ & 56 & 17.3 & 22.6 & 100.0 \\
\hline No response & 76 & 23.5 & 100.0 & \\
\hline
\end{tabular}

Table 2. Service Charges in the hospitals.

\begin{tabular}{lllll}
\hline Charges & Antenatal Clinic & Antenatal profile & Obstetric Ultra sound & Admission Charges \\
\hline Ksh. 330 & $23.5 \%$ & $23.5 \%$ & $0.0 \%$ & $0.0 \%$ \\
Ksh. 700 & $0.0 \%$ & $0.0 \%$ & $23.5 \%$ & $0.0 \%$ \\
Free & $75.5 \%$ & $76.5 \%$ & $76.5 \%$ & $100.0 \%$ \\
\hline
\end{tabular}

\subsection{State of Hospital Infrastructure}

The study established the shortage of Hospital infrastructure required for effective implementation and efficiency of free maternal healthcare services such as beds, infant incubators, toilets and bathroom and personnel midwives. This resulted to sharing of these resources as presented in Table 3.

Table 3. Availability and sharing of Hospital infrastructure by women seeking free maternal services.

\begin{tabular}{|c|c|c|c|c|c|}
\hline Infrastructure & Validity & Frequency & $\%$ Frequency & $\%$ Validity & $\%$ Cumulative \\
\hline \multirow{2}{*}{ Beds } & Yes & 244 & 75.3 & 75.3 & 75.3 \\
\hline & No & 80 & 24.7 & 24.7 & 100.0 \\
\hline \multirow{2}{*}{ Incubators } & Yes & 232 & 71.6 & 71.6 & 71.6 \\
\hline & No & 92 & 28.4 & 28.4 & 100.0 \\
\hline \multirow{2}{*}{ Availability of warm water for shower } & Yes & 8 & 2.5 & 2.5 & 2.5 \\
\hline & No & 316 & 97.5 & 97.5 & 100.0 \\
\hline \multirow{2}{*}{ Availability of Bed nets } & Yes & 184 & 56.8 & 56.8 & 56.8 \\
\hline & No & 140 & 43.2 & 43.2 & 100.0 \\
\hline
\end{tabular}

Over $244(75.3 \%)$ respondents indicated that they had shared bed. At the same time, there was evidence of sharing incubators with $232(71.6 \%)$ of the respondents revealing that their children shared an incubator. Majority of the women $316(97.5 \%)$ did not have hot shower and those provided was under personal request to midwife on duty. Although majority of women $184(56.8 \%)$ were provided with bed nets, $140(43.2 \%)$ provided the evidence that bed nets were not enough. 


\subsection{Condition of Maternity Wards in the Hospitals}

The cleanliness of wards was ranked as fairly by $61.7 \%$, as good by $25.9 \%$ and as poor by $12.3 \%$. The general cleanliness of toilet and bathroom was rated by $61.7 \%$ as fair, as good by $17.3 \%$ and as poor by $21 \%$. Some of the respondents indicated that blood soaked cotton wool was not properly disposed hence the need to provide dustbins in the toilet.

Table 4. Cleanliness of Maternity Wards, toilets and bathrooms.

\begin{tabular}{|c|c|c|c|c|c|}
\hline Cleanliness & Validity & Frequency & \%Frequency & \%Validity & $\%$ Cumulative \\
\hline \multirow{3}{*}{ Maternity Wards } & Poor & 40 & 12.3 & 12.3 & 12.3 \\
\hline & Fair & 200 & 61.7 & 61.7 & 74.1 \\
\hline & Good & 84 & 25.9 & 25.9 & 100.0 \\
\hline \multirow{3}{*}{ Toilets and bathrooms } & Poor & 68 & 21.0 & 21.0 & 21.0 \\
\hline & Fair & 200 & 61.7 & 61.7 & 82.7 \\
\hline & Good & 56 & 17.3 & 17.3 & 100.0 \\
\hline
\end{tabular}

\subsection{Nature of Human Resource in the Hospitals}

The relationship between human resource and the implementation of free maternal healthcare services was also studied by looking at staffing size and staff attitude, courtesy and promptness (average response time) in duty attendance. The current study established that that midwives were not enough as agreed by $208(64.2 \%)$ of the respondents while $116(35.8 \%)$ agreed that nurses/ midwives were adequate. Although the staffing size was small, there was demonstrated evidence of reliability at $56.8 \%$ but low cooperativity of $37 \%$ and good average response time. However, an element of hostility was also demonstrated as agreed by $6.2 \%$ of the respondents.

Table 5. Staff size and their attitude, courtesy and average response time in the hospital.

\begin{tabular}{llllll}
\hline & Validity & Frequency & \%Frequency & \%Validity & \%Cumulative \\
\hline \multirow{2}{*}{ Size } & Yes & 116 & 35.8 & 35.8 & 35.8 \\
& No & 208 & 64.2 & 64.2 & 100.0 \\
& Cooperative & 120 & 37.0 & 37.0 & 37.0 \\
Attitude and courtesy & Reliable & 184 & 56.8 & 56.8 & 93.8 \\
& Hostile & 20 & 6.2 & 6.2 & 100.0 \\
\multirow{3}{*}{ Average response time } & Less than 5 minutes & 24 & 7.4 & 7.4 & 7.4 \\
& $10-15$ minutes & 192 & 59.3 & 59.3 & 66.7 \\
& More than 15 minutes & 108 & 33.3 & 33.3 & 100.0 \\
\hline
\end{tabular}

\subsection{Source of Information and Dissemination on Implementation of Free Maternal Healthcare Services}

There was poor sources and dissemination of information on pregnant women on free maternal healthcare services being offered in the hospitals (Table 6). These included through relatives (38.3\%), during antenatal clinic visits (34.6\%) and service charter (14.8\%).

Table 6. Source of information and dissemination regarding provision of free maternity services.

\begin{tabular}{|c|c|c|c|c|c|}
\hline Parameter & Valid & Frequency & \%Frequency $t$ & $\%$ Valid & $\%$ Cumulative \\
\hline \multirow{4}{*}{ Source of information } & Relatives & 124 & 38.3 & 38.3 & 38.3 \\
\hline & Self & 40 & 12.3 & 12.3 & 50.6 \\
\hline & Mass media & 48 & 14.8 & 14.8 & 65.4 \\
\hline & ANC clinic & 112 & 34.6 & 34.6 & 100.0 \\
\hline \multirow[b]{2}{*}{ Information dissemination } & Yes & 52 & 16.0 & 16.3 & 16.3 \\
\hline & No & 268 & 82.7 & 83.8 & 100.0 \\
\hline
\end{tabular}

\subsection{Patient Satisfaction with Provision of Free Maternal Healthcare Services}

The study sought to establish patient satisfaction on the implementation of free maternal healthcare services offered in public hospitals in Kenya. There was good promptness $(61.7 \%)$ in terms of services delivery, good staff helpfulness $(64.2 \%)$ and fare Patient reception (56.2\%). However, 
elements of lack of patient satisfaction with provision of free maternal healthcare services offered in by the hospitals was

reported (Table 7).

Table 7. Patient Satisfaction with Provision of Free Maternal Healthcare Services.

\begin{tabular}{llllll}
\hline Element of staffs & Valid & Frequency & \%Frequency & \%Validity & \%Cumulative \\
\hline \multirow{4}{*}{ Patient reception } & Poor & 4 & 1.2 & 1.2 & 1.2 \\
& Fair & 184 & 56.8 & 56.8 & 58.0 \\
& Good & 112 & 34.6 & 34.6 & 92.6 \\
& Excellent & 24 & 7.4 & 7.4 & 100.0 \\
Helpfulness & Fair & 92 & 28.4 & 28.4 & 92.4 \\
& Good & 208 & 64.2 & 64.2 & 100.0 \\
& Excellent & 24 & 7.4 & 2.4 & 2.5 \\
promptness & Poor & 8 & 2.5 & 29.6 & 32.1 \\
& Fair & 96 & 29.6 & 61.7 & 93.8 \\
\hline
\end{tabular}

\section{Discussion}

The study targeted a sample size of 398 women who had given birth in Machakos level 5 hospitals in Machakos County from which 324 questionnaires were filled and returned accounting to a response rate of $81.4 \%$. This response rate was excellent and representative as Mugenda and Mugenda (1999) advocate for a response rate of 50\% [8]. According to Mugenda and Mugenda (1999), a response rate of $50 \%$ is adequate for analysis and reporting, rate of $60 \%$ is good while $70 \%$ and over is considered excellent.

As revealed by the demographic data, the study shows that all women irrespective of their age group, marital status, and level of education, occupation and average monthly income benefited from free maternal healthcare services at Machakos level 5 hospital. The finding of this research revealed that the average age of respondents who benefited from free maternal healthcare services was between 21-30years. This is in agreement with Lubbock and Stephenson (2008) findings on a study conducted in Nicaragua that the average age of women utilizing antenatal services was 26 years [9]. While majority were self-employed or unemployed, in a study on determinants of free maternal healthcare studies have found a correlation between average monthly house hold income and utilization of free healthcare services [10].

Resources are required for implementing free maternal healthcare services in public hospitals and are categorized into human resource and the financial resources. There is a direct relationship between resource allocation and implementation of free maternity services. Insufficient or slow distribution of the funds that are available for free maternity program could also create problems. According to Bosire (2013) some facilities have reportedly been given extra money to cover the influx of deliveries whereas others have remained in limbo, uncertain of how to balance the new policy for free care with their need to cover costs [11]. In countries like Ghana that have implemented free delivery service, findings have documented that funding and implementation gaps can create serious friction between health staff and communities and between facility managers and higher levels of the health system [7]. Underfunding can cause implementation failure of the policy leading to out-ofpocket payments for 'free' services. For instance, in Tanzania $73.3 \%$ of women with facility delivery reported having made out-of-pocket payments for delivery-related costs after abolition of user fees [12].

Although the maternal healthcare services are free, lack of infrastructure can make it costly as women may seek other services attached to it elsewhere. Infrastructure is important in the implementation of free maternal healthcare services. The finding of the study showed that there is a recognizable relationship between hospital infrastructure and implementation of free maternity services in public hospitals. This is reflected in most government hospitals around the country. The existing government hospitals were either built during colonial period or in the late 1970's and most of which have not been expanded to match the increasing population.

The $75.3 \%$ of the respondents shared bed, while $71.6 \%$ of the infants shared incubators. These findings were similar to those of Alberts et al, 2013 in Ghana which found that infrastructure of health facilities was not matching with the growing population [13]. From the observation of this study, the government through the Ministry of Health needs to equip the hospitals with equipment such as ultrasound machines and also ensure that Laboratories have enough reagents to run antenatal profile without subject women to pay for these services from the private hospital to realize the implementation of free maternal healthcare services. Expansion of the existing hospitals is crucial to cater for the increasing population to avoid sharing of crucial resources such as incubators and beds and also provide good sanitation. The study has shown the hospitals do not have enough midwives hence the need to employ more to cope with the growing number of women seeking healthcare services. Additionally, the government through the Ministry of Health needs to provide more funds to buy or replenish consumables in hospital providing free maternal healthcare services.

According to Kenya health sector strategic and investment plan (2008-12). It estimates that current staffing levels only meet $17 \%$ of the minimum requirements needed for effective operation of healthcare system. Kenya has only 7 nurses per 4000 residents and the ration of midwives to patients stand at 
1:20 against WHO recommendation of $1: 1$ for critical patients, 1:4 for stable patients. Healthcare is a people intensive activity, effective management of human resource for human resource for health aims at ensuring that adequate numbers of appropriately skilled and motivated workers are available to deliver free maternity services. The problem of inadequate staffing has further been enhanced by the introduction of free maternity services in public hospital. Hospitals have reported increased overcrowding at maternity wards for instance Kenyatta National Hospital in May 2013 reported 938 deliveries as compared to 1375 in May 2014 this increase was occasioned by introduction of free maternity services in all public hospitals in Kenya. These findings compare with findings on a study on exemption policy in Ghana which confirmed that introduction of free delivery care policy is associated with an increase in facility deliveries [14].

However, Nurses have also reported being overburdened due to the implementation of the new policy with one nurse attending to more than ten women a ratio of $1: 10$. In order to meet numerical staffing requirement the government ought to ensure that staff are used optimally and a number of human resource policies and practices are out in place. These policies need to include performance management, training and development through continuous professional development and it's in this note that the government need to allocate more resource to hire and train more midwives to be deployed in government hospitals.

The sources of information and its dissemination for women on is crucial implementation of free maternal healthcare services in public hospitals. Awareness was sighted as a major structural variable that could influence decision of women to deliver in public hospitals, such knowledge should highlight on requirement and various point of service delivery. It is therefore important for the government to put in place mechanisms that will regularly inform the general public and specifically pregnant women to deliver in public hospitals in Kenya. The study therefore establishes a positive and significant relationship between patient level of awareness and utilization of free maternal healthcare services. This study revealed that majority of the respondents hard about free maternal healthcare services during political campaign and while attending to antenatal clinic in public hospitals. In some studies, indicates that awareness is a structural variable that could influence utilization of free maternity services in public hospitals. Lack of awareness could be a major barrier to utilization of free services $56 \%$ of respondents who participated in this research got the information from the hospitals while attending prerequisite antenatal clinic (ANC) and through service charter displayed in all public hospitals. Mass media accounted for $14.8 \%$ where respondents referred to 2013 political campaigns by the Jubilee coalition as the source of information.

Patient satisfaction is also very important in the implementation of free maternal healthcare services in public hospitals. The study established that there is a positive and significant relationship between patient satisfaction implementation of free maternal healthcare services. Quality of service is attributed to skilled personnel who adhere to professional ethics. According to information gathered in this study $76.5 \%$ of the respondents were satisfied with services offered in public hospitals. In order to correct the misconception of women in relation to quality of services in public hospital, healthcare professionals need to adhere to the concept of caring and other humanistic attributes such as competence, confidence and compassion. Midwives also to improve on how they handle patients and this should focus on respect to religious beliefs traditions, culture and also provide feasible advice in relation to safe motherhood.

Patient Satisfaction and Implementation of Free Maternity Services in Public was pegged on the concept of caring, humanistic attribute of competence, confidence and compassion. Majority of the respondents talked of respect to their religious belief, culture where midwives or healthcare professional ought to observe these issues. The $57 \%$ of the respondents said they were received well, $64 \%$ indicated that midwives were prompt in attending to their healthcare needs while $62 \%$ said nurses were helpful. Matua (2004) reported that women who had gone to deliver in public hospitals had report negative attitude of healthcare providers [15]. He said that women are sometimes reluctant to use maternity services in public hospitals due to the fact that some midwives/Nurses are said to be rude, insensitive and threatening mothers that they will be left to deliver without assistance. On waiting time $59 \%$ of the respondents indicated that it took midwives approximately 10 - 15 minutes to attend to them while $33 \%$ said it took the more than 15 minutes to attend to them. Time taken for response may be attributed to increased number of women who deliver in public hospital since the introduction of free maternity services in public hospitals, as much as the government is rolling out this policy quality of services need not to be compromised so that many more women may have the confidence of delivering under the care of trained professional. Quality of healthcare is influenced by the capacity to use available input to deliver a desired outcome. User's satisfaction with care has increasingly been recognized as an important outcome of care. It is considered as a means of secondary prevention of maternal mortality since satisfied women may likely utilize the services optimally [16, 17]. A focus on quality of free maternal healthcare services will ensure that there is immediate response to clients. Quality may be affected by soft inputs such as care, health workers' attitude, motivation.

\section{Conclusion and Recommendations}

\subsection{Conclusions}

The study revealed that there is a significant relationship between hospital infrastructure and implementation of free maternal healthcare services in Kenya. Investment in infrastructure will ensure that the increasing numbers of women seeking to deliver in public hospitals is taken care of 
and issues of bed and incubator sharing do not take place in public hospitals. The study also revealed that there is a direct relationship between resources and implementation of free maternity services in public hospitals. The study identified that there were few Midwives/Nurse in public hospitals and more resources are required to ensure that staffing need are well taken care of. The study also deduced that there is a relationship between source of information and utilization of free maternity services in Kenya. Failure by the government to inform women on free maternity services may result into under-utilization of free services in lower level hospitals leading to overcrowding in some hospitals like $\mathrm{KNH}$ and Pumwani maternity hospital. The study also revealed that there is a direct relationship between patient satisfaction and utilization of free maternal healthcare services in public hospitals, hence the government need to employee best strategies that will ensure that patient receive the best services in public hospitals where patients are handled with care respect and professionalism. $78 \%$ of the respondents rated the quality of services as good hence the best pointer that patients are satisfied with free maternal health services.

\subsection{Recommendations}

The study finding unveiled a number of suggestions concerning implementation of free maternal healthcare services in public hospitals. The following are therefore recommendations on the findings: The government allocates more recourses to ministry of health to help address the issues of human resource and equipment gaps in government hospitals. The Ministry of health to review staffing needs in public hospitals. To identify the state of equipment and needs in public hospitals in Kenya. Ministry of Health to encourage continuous dissemination of information regarding free maternal healthcare services in public hospitals. The government put more emphasis on Patient satisfaction as a way of attracting more patients to deliver in public hospitals. Ministry of health need to address soft inputs such as patient care and health workers' attitude towards patients. There may be a need for further research to determine the quality of services after the introduction of free maternal healthcare services in public hospitals in Kenya.

\section{Acknowledgement}

I would like to express my special thanks to my teacher Dr. Kipruto Hillary for the support he gave me in conceptualizing the data collection and management framework. To my parents, Mr. and Mrs. Charles Moemi for the financial and moral support the offered during the course of this study. God, bless you abundantly.

\section{References}

[1] Esipisu I, 2011. Gadam sorghum in semi-arid Eastern Kenya. Http://ipsnews.net/news.asp?idnews=55737 Sorghum Proving Popular with Kenyan Farmers.
[2] Griffins and Stevenson (2001), Utilization of Maternal Healthcare Services in India, Understanding regional differences. University of Maryland, USA.

[3] Gage, A. J. (2007). Barriers to the utilization of maternal health care in rural Mali. Social Science \& Medicine, 65 (8), 1666-1682.

[4] Bruce J. (1990). Fundamental elements of the quality of care: a simple framework. Studies in Family Planning 21 (2): 6191.

[5] The World Health Organization. 2011. The Abuja Declaration: Ten years on. Retrieved from http://www.who.int/healthsystems/publications/abuja_report_a ug_2011.pdf.

[6] Nicole Bourbonnais, 2013. Implementing free maternal health care in Kenya; Challenges, strategies and recommendations. KNCHR 6, November 2013.

[7] Johnson B. and Onwuegbuzie J. A. Mixed Methods Research: A Research Paradigm Whose Time Has Come. Health Policy and Planning 2007; 22: 167-177 doi: 10.1093/heapol/czm008.

[8] Mugenda, O. M. and Mugenda, A. G. (1999) Research Methods: Quantitative and Qualitative Approaches. Acts Press, Nairobi.

[9] Lubbock, L. A, Stephenson, R. B. Utilization of Maternal health care services in the department of Malagalpa, Nicaragua. Rev. Pnanam Salud Publica. 2008: 24 (2): 75-84.

[10] Shariff, A. and Singh, G (2002). Determinants of maternal health care utilization in India: Evidence from a recent household survey. NCAER, Working Paper Series No. 85. New Delhi: National Council of Applied Economic Research.

[11] Bosire Boniface, "Kenyan Hospitals Slow to Comply with Waived Maternity Fee Directive," Sabahi, June 5, 2013, http://sabahionline.com/en GB/articles/hoa/articles/features/2 013/06/05/feature-02.

[12] Kruk M, Mbaruku G, Rockers P \& Galea S. User fee exemptions are not enough: out-of-pocket payments for "free" delivery services in rural Tanzania. Tropical Medicine and International Health. 2008; 13 (12): 1442-51.

[13] Albert, A, Anita, A. D, and Kwabena A. P, 2013. Factors Influencing the Utilization of Maternal Health Services: The Perspective of Rural Women in Ghana. Journal of Public Administration and Governance, ISSN 2161-7104 2013, Vol. 3, No. 2.

[14] Dzakpasu S, Soremekun S, Manu A, Asbroek G, Tawiah C, et al. Impact of Free Delivery Care on Health Facility Delivery and Insurance Coverage in Ghanas Brong Ahafo Region. PLos ONE. 2012; 7 (11), E49430. PubMed | Google Scholar.

[15] Matua, A. G., 2004. Determinants of maternal choices for place of delivery in Ayiru county, Uganda. Africa Journal of Nursing and Midwifery 6 (1): 33-38.

[16] Bazant ES, Koenig MA. Women's satisfaction with delivery care in Nairobi's informal settlements. Int J Qual Health Care 2009 Apr; 21 (2): 79-86.

[17] Lwanga SK. Sampling techniques in collection of health statistics in developing countries. East Afr Med J 1970 Apr; 47 (4): 202-11. 